\title{
A 46-year-old Chinese woman presenting with retroperitoneal follicular dendritic cell sarcoma: a case report
}

Taize Yuan, Qiuxiang Yang, Huanhuan Zhang, Jian Li and Xiuping Zhang*

\begin{abstract}
Introduction: Follicular dendritic cells are non-phagocytic, non-lymphoid cells of the immune system that are necessary for antigen presentation and the regulation of reactions in the germinal centers of the lymph nodes. Follicular dendritic cell sarcoma is an unusual cancer, particularly in the intra-abdominal region. In the present report we describe an unusual case of retroperitoneal follicular dendritic cell sarcoma that emphasizes the difficulty of diagnosing and treating this tumor. Retroperitoneal follicular dendritic cell sarcoma has only been rarely reported in the literature to date.

Case presentation: A 46-year-old Chinese woman of Han ethnicity presented with chronic right lower quadrant abdominal pain over the preceding 4 weeks. The tumor was resected and submitted to histopathological examination. The case was verified as retroperitoneal follicular dendritic cell sarcoma by microscopic examination and immunohistochemical analysis. After diagnosis, she received postoperative radiotherapy and chemotherapy. She has survived 3 years postoperatively, although she has a pulmonary metastasis.
\end{abstract}

Conclusions: Retroperitoneal follicular dendritic cell sarcoma may demonstrate aggressive potential. This study indicated that postoperative adjuvant radiotherapy and chemotherapy could extend the survival of a patient with retroperitoneal follicular dendritic cell sarcoma.

Keywords: Follicular dendritic cell sarcoma, Immunohistochemistry, Pathology, Retroperitoneal, Therapy

\section{Introduction}

Follicular dendritic cells (FDCs) are among the accessory cells of the lymphoid system. Their main function is to trap and present antigens and immune complexes to B cells. FDCs are found mainly in the germinal centers of the lymphoid tissue, where they form a tight meshwork. FDCs feature complement receptors and human leukocyte antigen-DR on their surfaces [1,2]. Lymphoid follicles are found in the lymph nodes and in extranodal lymphoid tissue [3]. Follicular dendritic cell sarcoma (FDCS), which was first reported by Monda et al. [1] in 1986, is a rare neoplastic proliferation that exhibits the morphological and immunophenotypic features of FDCS. To date, approximately 106 cases of FDCS have been reported in the literature, of which 46 occurred intra-abdominally [4]. We

\footnotetext{
*Correspondence: xiupingzhang67@163.com

Department of Radiation Oncology, Affiliated Tumor Hospital, Guangzhou Medical University, No.78 Hengzhigang, Road, Guangzhou, Guangdong
} Province 510095, China report a case with retroperitoneal FDCS that emphasizes the difficulty of diagnosing and treating this tumor.

\section{Case presentation}

A 46-year-old Chinese woman of Han ethnicity presented with a 4-week history of chronic right lower quadrant abdominal pain without other associated symptoms. A general systemic examination was normal except for a large mass in her right lower quadrant. There was no significant past medical history. Routine biochemical and hematological tests were within normal limits. A chest X-ray also revealed no abnormalities. A subsequent abdominal computed tomography (CT) scan revealed a $8.6 \times 6.0 \mathrm{~cm}$ oval-shaped mass, with a clear boundary and mild-moderate enhancement, in her lower right retroperitoneal region, pressing against her right psoas muscle and her right iliac artery (Figure 1A).

She underwent an operation to resect the mass. Surgical exploration revealed a retroperitoneal mass measuring 

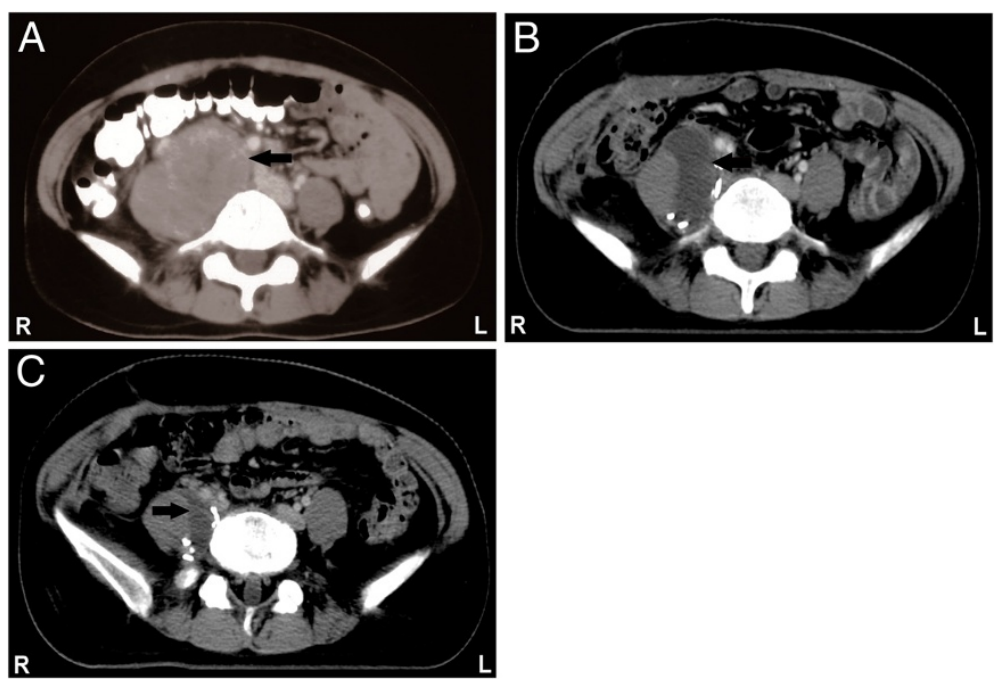

Figure 1 Computed tomography images of retroperitoneal follicular dendritic cell sarcoma. A: Contrast-enhanced computed tomography scan of the abdomen revealing an $8.6 \mathrm{~cm}$ retroperitoneal mass (arrow); B: liquid dark area (arrow) observed in the surgical area after operation; C: significant shrinking of the liquid dark area (arrow) was observed after adjuvant therapy.

$9 \times 6 \mathrm{~cm}$ adjacent to her transverse colon, her right psoas muscle and her vertebral column and covering her inferior vena cava. No associated lymphadenopathy was observed. Because the mass had infiltrated her right psoas muscle and was attached to her right iliac artery and her inferior vena cava, most of the gross tumor was resected, and the margin was positive. A CT scan revealed a liquid dark area in the surgical area after the operation (Figure 1B).

A cut section revealed a homogenous solid mass with a rough surface. Multiple sections were taken from various areas of the tumor. Microscopic examination revealed that the tumor was composed of fat to long spindle cells with multinodular infiltration that were arranged in a storiform pattern (Figure 2). The tumor exhibited necrosis, cell

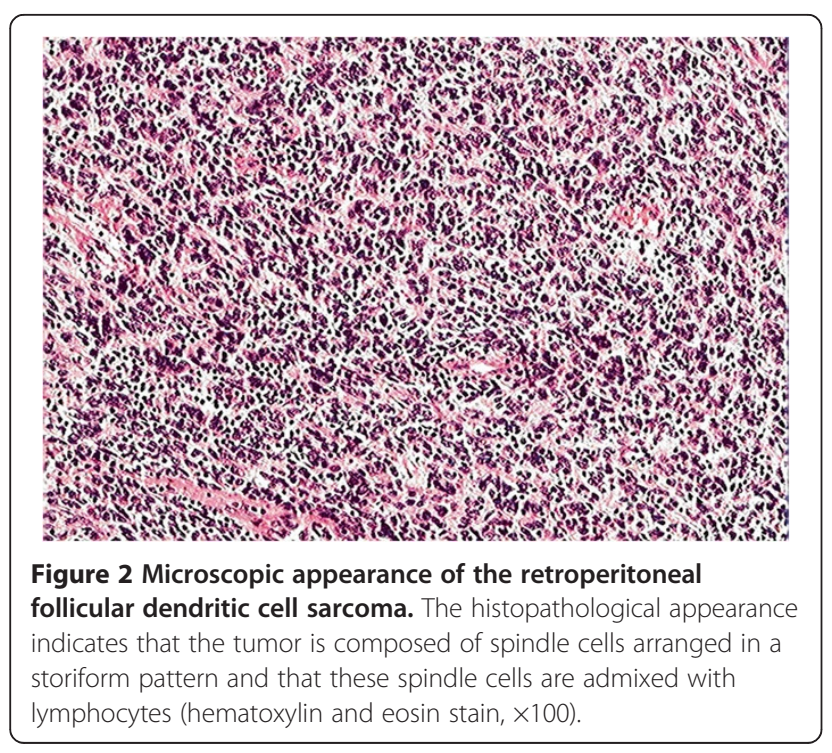

atypia and obvious mitosis. The mitotic events numbered up to 12 per 10 high-powered fields, and there was focally marked nuclear atypia, including pleomorphic, multinucleated cells. Furthermore, the tumor cells exhibited positive immunohistochemical staining for vimentin, CD21, CD35, D2-40 and leukocyte common antigen (Figure 3A to $3 \mathrm{E})$ as well as negative staining for cytokeratin (Figure 3F), CD20, CD30, CD117, epithelial membrane antigen and S-100 (not shown). Based on these histopathological and immunohistochemical findings, the patient was diagnosed with FDCS of the retroperitoneal lymph node.

She received postoperative sequential chemotherapy and radiotherapy. Three cycles of chemotherapy with a standard dose of cyclophosphamide, doxorubicin, vincristine and prednisone (CHOP) were initiated in July 2010. A total radiation dose of $60 \mathrm{~Gy}$ was then administered over 6 weeks, followed by another three cycles of chemotherapy with CHOP. To evaluate the efficacy of the adjuvant therapy, an abdominal CT scan was performed, which revealed that the liquid dark area had shrunk significantly (Figure 1C).

Hospital records indicate that the patient has reached 3 years of follow-up. Although she is alive, she has a right pulmonary metastasis.

\section{Discussion}

FDCS is a rare tumor that originates in the germinal centers of the lymph nodes in FDCS and has low malignant potential. Monda et al. [1] first described the malignant neoplasm in 1986. FDCS occurs mainly in adults; the average age is 47 years (14 to 77 years). The incidence rate is the same for men and women [5]. The most common 


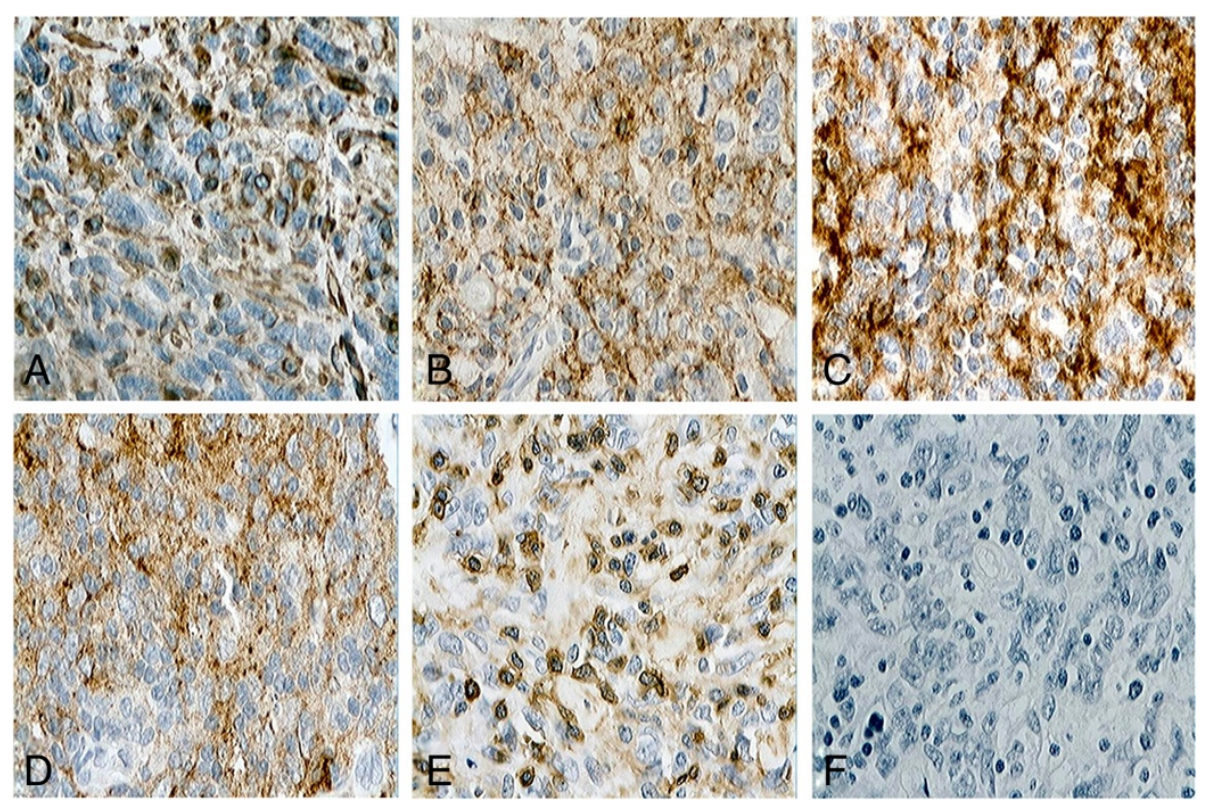

Figure 3 Immunohistochemical features of retroperitoneal follicular dendritic cell sarcoma (paraffin immunohistochemical stain $\times 400$ ). A: Positive immunohistochemical staining for vimentin; B: Positive immunohistochemical staining for CD21; C: Positive immunohistochemical staining for CD35; D: Positive immunohistochemical staining for D2-40; E: Positive immunohistochemical staining for leukocyte common antigen; F: Negative immunohistochemical staining for cytokeratin.

clinical symptom is a painless and slow-growing lymph node. FDCS is found mostly in the lymph nodes, particularly the cervical lymph nodes and occasionally in the axillary and mediastinal lymph nodes. FDCS is also occasionally found outside the lymph nodes. Extranodal FDCS has been found in the tonsils, nasopharynx, liver, spleen and gastrointestinal tract [3,6-9].

Pathological diagnosis is challenging [10] and may require a combination of morphological, immunophenotypical, cytochemical and electronmicroscopic analyses [2]. FDCS may be suspected if the tumor exhibits distinct microscopic features, such as a storiform arrangement of spindle-shaped cells, indistinct cell borders and a background of lymphocytes scattered throughout the neoplastic cells [11]. The morphology of our case is typical of FDCS, although various studies have reported atypical presentations in the form of a multifocal disease, increased mitosis, giant cell transformation and wide areas of necrosis. To confirm the tumor, immunohistochemical staining is required to detect the most widely used FDC markers, including CD21, CD35, vimentin and D2-40, which can distinguish FDCS from other spindle cell neoplasms [11,12]. S-100 and certain vascular or muscular markers may also help to distinguish this tumor from other tumors, such as malignant peripheral nerve sheath tumors and gastrointestinal stromal tumors [13].

A complete surgical excision is the appropriate initial therapy [3]. Adjuvant radiotherapy or chemotherapy appears to be indicated in cases of incompletely resected lesions or adverse pathological features during recurrence [14]. However, because of the rarity of the lesion and the retrospective nature of the published literature, the role of adjuvant therapy (radiotherapy or chemotherapy) remains unclear. A few recent reports have suggested that FDCS should be viewed as an intermediate-grade malignancy that presents a substantial risk of metastasis to the lung, liver, intra-abdominal soft tissues and lymph nodes $[11,15]$.

\section{Conclusions}

As in this patient, retroperitoneal FDCS may demonstrate aggressive potential. This study indicated that postoperative adjuvant radiotherapy and chemotherapy could extend the survival of a patient with retroperitoneal FDCS.

\section{Consent}

Written informed consent was obtained from the patient for publication of this case report and accompanying images. A copy of the written consent is available for review by the Editor-in-Chief of this journal.

\section{Abbreviations}

CHOP: Cyclophosphamide, doxorubicin, vincristine and prednisone; CT: Computed tomography; FDCs: Follicular dendritic cells; FDCS: Follicular dendritic cell sarcoma.

\section{Competing interests}

The authors declare that they have no competing interests. 


\section{Authors' contributions}

TY and QY contributed equally to this work; TY, QY and XZ analyzed and interpreted the data from our patient and drafted the manuscript. $\mathrm{HZ}$ and $J \mathrm{~L}$ performed the histological examination of the tumor. All authors read and approved the final manuscript.

\section{Acknowledgements}

We acknowledge our patient for allowing us to publish this case report.

Received: 3 December 2013 Accepted: 5 February 2014

Published: 3 April 2014

\section{References}

1. Monda $L$, Warnke R, Rosai J: A primary lymph node malignancy with features suggestive of dendritic reticulum cell differentiation. A report of 4 cases. Am J Pathol 1986, 122:562-572.

2. Chan JK, Fletcher CD, Nayler SJ, Cooper K: Follicular dendritic cell sarcoma. Clinicopathologic analysis of 17 cases suggesting a malignant potential higher than currently recognized. Cancer 1997, 79:294-313.

3. Karabulut B, Orhan KS, Guldiken Y, Dogan O: Follicular dendritic cell sarcoma of the nasopharynx. Int J Oral Maxillofac Surg 2012, 41:218-220.

4. Li L, Shi YH, Guo ZJ, Qiu T, Guo L, Yang HY, Zhang X, Zhao XM, Su Q: Clinicopathological features and prognosis assessment of extranodal follicular dendritic cell sarcoma. World I Gastroenterol 2010, 16:2504-2519.

5. Kairouz S, Hashash J, Kabbara W, McHayleh W, Tabbara IA: Dendritic cell neoplasms: an overview. Am J Hematol 2007, 82:924-928.

6. Low SE, Menasce LP, Manson CM: Follicular dendritic cell sarcoma: a rare tumor presenting as an abdominal mass. Int I Surg Pathol 2007, 15:315-317.

7. Sander B, Middel P, Gunawan B, Schulten HJ, Baum F, Golas MM, Schulze F, Grabbe E, Parwaresch R, Fuzesi L: Follicular dendritic cell sarcoma of the spleen. Hum Pathol 2007, 38:668-672.

8. Martins PN, Reddy S, Martins AB, Facciuto M: Follicular dendritic cell sarcoma of the liver: unusual presentation of a rare tumor and literature review. Hepatobiliary Pancreat Dis Int 2011, 10:443-445.

9. Eun YG, Kim SW, Kwon KH: Follicular dendritic cell sarcoma of the tonsil. Yonsei Med J 2010, 51:602-604

10. Malik A, Veniyoor A, Fanthome B, Dutta V: Follicular dendritic cell sarcoma: a diagnostic challenge. J Cancer Res Ther 2012, 8:306-307.

11. Shia J, Chen W, Tang LH, Carlson DL, Qin J, Guillem JG, Nobrega J, Wong WD, Klimstra DS: Extranodal follicular dendritic cell sarcoma: clinical, pathologic, and histogenetic characteristics of an underrecognized disease entity. Virchows Arch 2006, 449:148-158.

12. Yu H, Gibson JA, Pinkus GS, Hornick JL: Podoplanin (D2-40) is a novel marker for follicular dendritic cell tumors. Am J Clin Pathol 2007, 128:776-782.

13. Long-Hua Q, Qin X, Ya-Jia G, Jian W, Xiao-Yuan F: Imaging findings of follicular dendritic cell sarcoma: report of four cases. Korean J Radiol 2011, 12:122-128.

14. Mondal SK, Bera H, Bhattacharya B, Dewan K: Follicular dendritic cell sarcoma of the tonsil. Natl J Maxillofac Surg 2012, 3:62-64.

15. Fareed MM, Memon MA, Rashid A, Furrukh M, Ahmed S, Ghouri AR, Khan A, Asghar AS: Follicular dendritic cell sarcoma of the neck with pulmonary metastases. J Coll Physicians Surg Pak 2011, 21:561-563.

\section{doi:10.1186/1752-1947-8-113}

Cite this article as: Yuan et al: A 46-year-old Chinese woman presenting with retroperitoneal follicular dendritic cell sarcoma: a case report. Journal of Medical Case Reports 2014 8:113. 